\title{
ETUDES SOCIO-ECONOMIQUES : Le protocole biosécurité : entre incertitude et ambiguïté
}

\section{SOCIO-ECONOMIC STUDIES: The biosafety protocol: between uncertaintly and ambiguity}

Oléagineux, Corps Gras, Lipides. Volume 7, Numéro 4, 350-2, Juillet - Août 2000, Dossier : "OGM: expertise et décision publique"

Auteur(s) : Andrée SONTOT, Ecole doctorale Sciences-Po CERI, 56, rue Jacob, 75006 Paris.

Résumé : Le 29 janvier 2000 a été adopté à Montréal le protocole de Cartagena sur la prévention des risques biotechnologiques relatif à la convention sur la diversité biologique. Si les travaux de la convention sur la diversité biologique suscitent généralement peu l'intérêt du grand public, l'adoption de ce protocole a été reconnue comme un fait marquant tant par la presse, par de nombreuses organisations non gouvernementales et des leaders d'opinion, que par des responsables gouvernementaux. Cet intérêt s'explique probablement autant par la complexité des enjeux qui y sont liés, qui concernent potentiellement une grande diversité d'acteurs, que par la difficulté de la négociation, qui a contribué à accroître encore la sensibilité des uns et des autres sur les questions débattues. Pourtant, paradoxalement, chacun semblait satisfait du résultat obtenu, au point d'afficher sa victoire sur les points qui lui paraissaient essentiels. Avec quelques mois de recul, on peut désormais poser la question plus sereinement : ce protocole est-il un bon compromis, un marché de dupes, ou une avancée pratique mais limitée ?

Mots-clés : GMO, biodiversity, environment and trade, expertise, precautionary principle.

Summary: The adoption of the Cartagena Protocol on Biosafety on January 29th 2000 closed a long and difficult international negociation on the prevention of biotechnological risks on the environment. If some of the issues opposed developed and developing countries, the main problems were trade-related and opposed the European Union to the "Miami group" lead by the US. The final agreement is a compromise text, whose main interest it to provide a common framework for the international management of biosafety, especially through risks evaluation. Scientific expertise will play an important role in the implementation of the agreement.

Keywords: GMO, biodiversity, environment and trade, expertise, precautionary principle.

\section{ARTICLE}

\section{Une négociation difficile en quatre phases}

La négociation du protocole biosécurité a dû composer en permanence avec cinq problématiques :

- une problématique environnementale, autour du risque de flux de gènes incontrôlés dans l'environnement, susceptible de poser des problèmes de conservation de la diversité biologique ; 
- une problématique réglementaire, autour de la nécessité de donner au commerce des organismes génétiquement modifiés (OGM) un cadre international, alors que se développent les législations nationales sur ces produits et que se pose la question de leur compatibilité ;

- une problématique commerciale, autour de la question d'un éventuel régime dérogatoire à la règle générale pour les $O G M$;

- une problématique politique, autour de la relation entre Organisation mondiale du commerce (OMC) et accords multilatéraux sur l'environnement ;

- une problématique scientifique, autour du rapport entre expertise scientifique, acteurs économiques, et décision politique.

La question d'un texte international sur les questions de sécurité biologique apparaît dès 1988 sur l'agenda international, dans le cadre de la préparation du sommet de la terre de 1992 et de l'agenda 21. Elle oscille alors entre la seule biotechnologie, le lien entre biotechnologie et biodiversité, la santé et la sûreté humaine. Le débat se déroule alors principalement entre certains pays européens, qui demandent un code de conduite ou un instrument juridique spécifique à la biotechnologie, et les États-Unis, qui considèrent que les produits issus de la biotechnologie peuvent être couverts par les instruments juridiques existants et que, la biotechnologie n'étant pas dangereuse par elle-même, il n'y a aucune raison de la réguler. Cette première phase du débat international débouchera sur l'article 19.3 de la convention sur la diversité biologique ${ }^{3}$ (encadré) : " les parties examineront s'il convient de prendre des mesures et d'en fixer les modalités, éventuellement sous forme d'un protocole, comprenant notamment un accord préalable donné en connaissance de cause définissant les procédures appropriées dans le domaine du transfert, de la manutention et de l'utilisation en toute sécurité de tout organisme vivant modifié résultant de la biotechnologie qui risquerait d'avoir des effets défavorables sur la conservation et l'utilisation durable de la diversité biologique ».

S'ensuit, entre 1992 et 1997, une phase de " cadrage " de la négociation assez confuse, essentiellement axée sur le format et les objectifs de la négociation à mener. Pour beaucoup de délégations, c'est aussi une phase de mise à niveau technique sur le sujet. Plusieurs éléments y apparaissent néanmoins, qui deviendront des constantes de la négociation : l'influence majeure d'une " non-partie » à la convention sur la diversité biologique (à savoir les États-Unis), à l'époque opposée au principe même de ce protocole, une faille entre pays en développement à revenu intermédiaire (en particulier d'Amérique latine) et pays les moins avancés (en particulier d'Afrique), la pression des ONG sur le débat ( 8 d'entre elles demandant en juillet 1996 un moratoire sur la distribution de tout produit et OGM jusqu'à la mise en place d'un protocole). 


\section{Le protocole biosécurité et la convention sur la diver sité biologique}

La convention sur la diversité biologique (CDB) adoptée à Rio le 5 juin 1992 est un accord-cad re définissant les principes généraux que do ivent appliquer ks états pa ities en matière de conservation de la diversité bio bgique, d'utilisation durable de seséléments, et de partage juste et équitable des avantages découlant de l'ex ploitation des ressoures génétiques. Le champ couvert par la CDB est très large, puisqu'il porte sur les tro is nivea ux d'organisation biobgique (diversité de séco systèmes, diversité entre espèces, et diversité génétiq ue au sein des espèces, tant végétales qu'animales et microbiennes), et un gra nd nombre de questions politiquement et économiquement très sensibles : l'accès aux ressoures génétiques et $k$ partage des avantages découlant de kur utilisation, la reconnaissance et la protection des savoirs traditionnels des communautés bcales et autochtones, le transfert de technologies, la gestion de a biotechnologie et la répa rition de ses avantages.

Cest dans le cadre de ce dernier point qứtait po sée la question d'une procédure d'accord préalable pour le transfert, la manutention et l' utilisation des organismes vivants modifiés pouvant avoir des effets défavorables sur la diversité biologique. La négociation de ce protocole * bio sécurité * s'est donc déroulée parallèlement aux débats sur kesautres points de la $\mathrm{CDB}$, et à ceux d'autres enceintes intema tionales traitant de questions connexes, comme $k$ commerce des matières prem ìres ag ricoles (Organisation mondiale du commere) ou le suivides orga nismes vivants modifiés da ns le doma ine alimentaire (Codex Alimentarius, dépendant de la FAO).

Le rythme s'accélère entre 1997 et 1998, avec l'élaboration des premières versions de texte, essentiellement autour d'un axe de débat nord-sud. Parmi les principales questions débattues, plusieurs émanent des pays en développement, comme celle des " produits dérivés " (sont-elles incluses ou non dans le champ ?), des considérations socio-économiques, de la responsabilité et de la réparation en cas de dommage, du trafic illicite. D'autres constituent plutôt des points de débat nord-nord : principe de précaution, non-discrimination commerciale.

Enfin, d'août 1998 à janvier 2000, se déroule la véritable négociation, essentiellement cette fois selon un axe nord-nord, comme en témoigne la liste des points d'achoppement les plus difficiles : commerce international, en particulier avec les parties non signataires, ou sur la question des matières premières issues d'OGM, rapport entre régimes de régulation domestiques (comme les réglementations communautaires en matière d'OGM) et internationaux. Autour de ces clivages se constituent cinq groupes de négociation : I'Union européenne, l'Europe centrale et orientale, le « groupe de Miami » (Argentine, Australie, Canada, Chili, Nouvelle-Zélande, États-Unis, Uruguay), le " groupe de même esprit " (regroupant la majorité des pays en développement), le " groupe de compromis " (Japon, Mexique, Norvège, Corée du Sud, Suisse). La sensibilité de l'opinion publique sur le sujet et la pression des ONG sur les gouvernements s'accroissent, particulièrement en Europe. Les difficultés rencontrées pendant la même période par d'autres négociations environnementales (climat) contribuent à la tension ambiante. La conférence extraordinaire des parties de Cartagène (Colombie) de février 1999, qui devait adopter le protocole, se soldera par un échec, à l'initiative du groupe de Miami et malgré le soutien de tous les autres groupes à la proposition « en paquet » de l'Union européenne.

Le succès obtenu à Montréal en janvier 2000 ne peut s'expliquer par les seules réunions plus ou moins formelles par lesquelles les délégations ont cherché à relancer le processus, ni par le talent unanimement reconnu de Juan Mayr, nouveau président de la conférence des parties extraordinaire en 1999-2000. II semble que les États-Unis aient sensiblement évolué par rapport à la convention sur la diversité biologique, le secteur privé y étant apparemment moins défavorable, et par rapport aux OGM. Un sondage réalisé fin 1998 [1] semble témoigner d'une évolution parallèle à celle de l'opinion européenne : " près des trois quarts s'opposent à ce que les 
compagnies brevettent le vivant. Autres résultats, $81 \%$ d'entre eux demandent un étiquetage des aliments modifiés par génie génétique, et $58 \%$ refuseraient de les acheter ". La distinction très claire opérée alors par l'administration américaine en matière d'OGM entre le domaine de l'environnement (le protocole biosécurité) et celui de l'alimentation (Codex Alimentarius) lui permet de répondre aux préoccupations environnementales exprimées par une partie de son opinion publique (particulièrement importante en période électorale) et de ses scientifiques sans mettre en cause directement le marché final de son secteur économique. L'Agence de protection de l'environnement américaine (Environmental Protection Agency) met ainsi en place en janvier 2000 des restrictions à la mise en culture de maïs et de coton génétiquement modifiés afin d'améliorer la gestion de la résistance des insectes au Bt. Enfin, l'échec de la conférence de Seattle fin 1999 a tué dans l'œuf la tentative de déplacer la négociation sur la biosécurité dans le cadre de I'OMC.

\section{Un contenu et un bilan ambigu}

Le champ d'application du protocole est défini par l'article $4:$ « le présent protocole s'applique aux mouvements transfrontières, au transit, à la manipulation et à l'utilisation de tout organisme vivant modifié (OVM) qui pourrait avoir des effets défavorables sur la conservation et l'utilisation durable de la diversité biologique, en tenant compte également des risques pour la santé humaine ». La fin de la phrase a donné lieu dans la presse à des interprétations très larges du champ du protocole. Dans le contexte de la convention sur la diversité biologique toutefois, la référence à la santé humaine ne porte pas sur la toxicité éventuelle de l'OVM en tant qu'aliment, mais sur la possible compétition de ce dernier avec les espèces locales, ce qui peut constituer une menace pour l'approvisionnement en denrées alimentaires. Le protocole porte donc bien sur la protection des risques biotechnologiques sur l'environnement, et non sur la santé humaine. Une nuance a néanmoins été introduite à la demande des pays en développement, autorisant les parties (art. 5) à demander une évaluation des risques des produits pharmaceutiques génétiquement modifiés destinés à la consommation humaine avant leur importation.

Le cœur du protocole est constitué par la description des procédures à suivre en matière d'échanges. Deux procédures principales sont prévues. La première, " procédure d'accord préalable en connaissance de cause " (AIA, advanced informed agreement), définie par les articles 7 à 10 et 12 , s'applique aux " organismes vivants modifiés destinés à être introduits dans l'environnement ", soit essentiellement les semences. Elle prévoit une notification par la partie exportatrice à la partie importatrice avant le premier mouvement transfrontière (art. 8), un accusé de réception de la notification (art. 9) et une procédure de décision complexe (art. 10).

La deuxième, sobrement qualifiée de " procédure à suivre ", porte sur les organismes vivants modifiés " destinés à être utilisés directements pour l'alimentation humaine ou animale ou à être transformés ", soit, plus simplement, les matières premières agricoles. Cette procédure consiste à communiquer au centre d'échange d'information créé par l'article 20 les décisions prises dans ce domaine pour leur utilisation à des fins nationales.

Le cas particulier de "l'utilisation en milieu confiné ", visant en fait la recherche, est reconnu par l'article 6, qui le dispense d'accord pré- alable en connaissance de cause, à condition que le mouvement transfrontière soit conforme aux normes de la partie importatrice. 
La réponse apportée par le protocole à la question de l'identification des OVM est plus ambiguë. En ce qui concerne les OVM « destinés à être utilisés en milieu confiné » et les " OVM destinés à être introduits intentionnellement dans l'environnement ", l'article 18 prévoit que chaque partie prend des mesures pour exiger que la documentation accompagnant ces OVM « indique clairement qu'il s'agit d'OVM ». En revanche, pour les " OVM destinés à être utilisés directement pour l'alimentation humaine et animale », il est prévu que la documentation les accompagnant " indique clairement qu'ils "peuvent contenir" des OVM ». Cette question, qui a constitué un point de blocage jusqu'à la dernière minute des négociations, constitue une concession majeure faite par I'Union européenne aux États-Unis. Compte tenu de ses propres ambitions en matière de traçabilité et d'étiquetage, l'Union européenne exigeait en effet une identification explicite des matières premières agricoles génétiquement modifiées faisant l'objet d'un mouvement transfrontière, avec identification de tous les OVM présents dans le chargement et numéro d'identification unique par OVM. Cette revendication induisait une ségrégation des matières premières agricoles à tous les stades (production, stockage, transport...) évidemment coûteuse pour la filière OGM. La formule " peut contenir des OVM ", si elle est utilisée systématiquement, ce qui est probable, pourrait obliger la filière traditionnelle à pratiquer l'étiquetage inverse " sans OVM » et à supporter le coût associé. Le compromis passé avec les États-Unis prévoit néanmoins la révision des modalités d'identification dans les deux ans suivant l'entrée en vigueur du protocole.

Compte tenu de la réglementation communautaire, il était essentiel pour l'Union européenne que les accords et arrangements bilatéraux, régionaux et multilatéraux soient reconnus par le protocole. Elle a obtenu un article 14 en ce sens.

Afin de permettre à tous les États d'appliquer pleinement le protocole quelles que soient leurs capacités scientifiques et administratives, un Centre d'échange pour la prévention des risques biotechnologiques est créé (article 20) auquel seront communiquées toutes les informations liées aux décisions des parties, en particulier concernant l'évaluation des OVM. Les parties sont également invitées à coopérer pour renforcer les capacités des pays en développement (art. 22).

Le point le plus marquant politiquement du protocole ne fait pas l'objet d'un article spécifique, ni d'ailleurs de la même interprétation des deux côtés de l'Atlantique. L'Union européenne se félicite de ce que le principe de précaution soit explicitement énoncé dans le corps des deux articles les plus opérationnels du protocole : 10.6 (accord préalable en connaissance de cause) et 11.8 (procédure à suivre pour les matières premières agricoles). "L'absence de certitude scientifique due à l'insuffisance des informations et connaissances scientifiques pertinentes concernant l'étendue des effets défavorables potentiels d'un OVM sur la conservation et l'utilisation durable de la diversité biologique dans la partie importatrice, compte tenu également des risques pour la santé humaine, n'empêche pas cette partie de prendre comme il convient une décision concernant I'importation de cet OVM pour éviter ou réduire au minimum ces effets défavorables potentiels. » Les États-Unis de leur côté, s'appuyant sur le préambule qui se réfère seulement à « l'approche de précaution ", peuvent affirmer ${ }^{4}$ que " les États-Unis n'ont avalisé en aucune façon le "principe de précaution" ».

L'autre point politiquement très important portait sur l'articulation de ce protocole avec les autres accords internationaux, particulièrement ceux liés au commerce. Le groupe de Miami affichait une position très dure, exigeant qu'une disposition stipule que rien dans le protocole n'affectait les 
droits et obligations liant les parties en vertu d'autres traités, celui instituant I'OMC étant évidemment particulièrement visé. L'Europe et la plupart des pays en développement considéraient qu'une telle disposition reviendrait à subordonner le protocole à l'OMC. Finalement, un triple considérant a été adopté en préambule, dont la formulation est soigneusement équilibrée pour donner satisfaction à toutes les parties. Le protocole n'a donc pas permis de régler clairement cette question.

\section{L'expertise au cœur des perspectives et enjeux à venir}

Certes, le protocole couvre un champ limité à la prévention des risques biotechnologiques sur la diversité biologique et il constitue un compromis, souvent ambigu dans sa formulation. Un point positif est néanmoins incontestable : un protocole sur les risques biotechnologiques a été adopté, et il l'a été dans le cadre d'une organisation des Nations unies consacrée à l'environnement et à son utilisation durable.

Il est encore difficile de définir précisément ce qu'il va changer, d'autant plus qu'il lui manque encore quelques mois et ratifications pour entrer en vigueur. Pour les opérateurs européens, ses conséquences les plus concrètes devraient apparaître via la révision en cours de la réglementation communautaire sur les OGM, qui intégrera évidemment la substance du protocole. Pour les pays en développement, la mise en place du centre d'échange et les initiatives permettant de renforcer leurs capacités constituent évidemment la priorité. II faut d'ailleurs insister sur le caractère essentiel de l'efficacité du fonctionnement de ce centre d'échanges, non seulement pour les pays en développement, mais pour la mise en œuvre effective du protocole lui-même, puisqu'elle contribuera aux prises de décision nationales.

Sur le plan international, l'adoption de ce protocole légitime la poursuite du débat sur l'impact des biotechnologies, dans le domaine alimentaire cette fois. II est significatif que le début de l'année 2000 ait connu un agenda international chargé sur ce point, avec notamment le Codex Alimentarius et l'atelier organisé par l'OCDE à Cambridge. Elle va, par ailleurs, amener les experts à réévaluer l'articulation de l'ensemble des accords internationaux sur des points communs, comme le principe de précaution.

Le deuxième point positif du protocole porte sur la mobilisation de l'expertise scientifique pour l'évaluation des risques. Le protocole est en effet conçu comme un instrument international permettant de gérer l'incertitude liée à l'utilisation des OVM. Une annexe au protocole prévoit que l'évaluation des risques des OVM pour la diversité biologique devra être réalisée " selon des méthodes scientifiques éprouvées et avec transparence et peut tenir compte des avis techniques et des directives des organisations internationales compétentes ". Cette disposition, toute logique qu'elle soit, présente toutefois un paradoxe : en multipliant les avis, elle est susceptible d'ajouter à l'incertitude scientifique une incertitude induite par la diversité des institutions concernées, de leurs capacités et approches scientifiques, et de leurs motivations. Ce paradoxe milite toutefois clairement pour que soient menées en France et en Europe les expertises sur l'impact potentiel des OGM sur la diversité biologique qui seront nécessaires s'il s'agit pour l'Europe de défendre devant I'OMC des décisions prises selon le principe de précaution en application du protocole de Cartagena. 
Notes

${ }^{1}$ Centre d'études et de recherches internationales.

${ }^{2}$ Bureau des ressources génétiques.

${ }^{3}$ Convention adoptée en juin 1992, regroupant désormais 172 États, les États-Unis ne l'ayant toujours pas ratifiée à ce jour.

${ }^{4}$ Catherine Wotecki, sous-secrétaire d'État à l'agriculture, à l'occasion du Codex Alimentarius, le 13 avril 2000.

\section{REFERENCES}

1. DEROIN P (1999). L'Amérique vide son sac. Biofutur, $186: 4$.

2. Site Internet de la convention sur la diversité biologique : http://www.biodiv.org 